\title{
Review
}

Masakazu Hara*

\section{Potential use of essential oils to enhance heat tolerance in plants}

https://doi.org/10.1515/znc-2019-0233

Received December 25, 2019; revised February 22, 2020; accepted March 19, 2020

Abstract: Isothiocyanates, monoterpenes, and leaf volatiles that are components of essential oils induce the expression of heat shock protein genes in plant systems. Here, the modes of heat shock responses induced by the essential oil compounds and their heat-tolerance-enhancing activities are described. Traditionally, green manure produced from essential-oil-containing plants has been used because such manure is thought to have beneficial effects in fertilizing, allelopathic, antibacterial, and animal-repellent activities. In addition to these effects, stress (especially heat stress)-tolerance-enhancing activities can be expected. Biostimulants containing such essential oils may be able to maintain the yield and quality of crops under increasing ambient temperatures. In this review, chemicals that enhance the heat tolerance of plants are designated as heat tolerance enhancers (HTLEs). Some essential oil compounds can be categorized as HTLEs available for biostimulants.

Keywords: heat shock protein; heat shock response; heat tolerance enhancers; isothiocyanates; monoterpenes.

\section{Introduction}

Essential oils are plant specialized metabolites having characteristic odors. They have been used as traditional herbs, perfumes, food seasonings, and beverage flavorings [1-3]. Essential oils have also been used as pharmaceuticals, such as anti-inflammatories, analgesics, sedatives, and spasmolytics $[4,5]$. Preservation and repellence are important purposes of essential oils because they have effective antimicrobial, insecticidal, and

*Corresponding author: Masakazu Hara, Research Institute of Green Science and Technology, Shizuoka University, 836 Ohya, Shizuoka, Shizuoka 422-8529, Japan, Phone: +81-54-238-5134 Fax:+81-54-238-5134, E-mail: hara.masakazu@shizuoka.ac.jp antifeedant activities [6-8]. In nature, essential oils affect the growth of plants that neighbor essential-oil-producing plants. Some of them are allelochemicals that inhibit seed germination $[9,10]$. Conversely, recent studies have proposed that volatile compounds contained in essential oils might provide benefits to plants growing in unfavorable environments [11-13].

Climate changes affect agricultural production in complex ways. It has been projected that yields of major crops may decrease due to local warming if the crops do not adapt to environmental changes [14-16]. Extreme heat waves severely inhibit plant growth and reduce world crop production $[17,18]$. Another opinion is that the ancestors of crop species may have had the potential to adapt to high ambient temperatures because angiosperms evolved during the warm climate periods after the Mesozoic era. However, the heat tolerance of present crops may be low because they were bred during the Quaternary ice ages. This may be a reason why the yields and qualities of today's crops decrease in elevated temperatures. According to physiological experiments, even plants growing at high latitudes can survive in high-temperature environments after they become acclimated [19]. This supports the idea that any crop species can bear high ambient temperatures if their heat tolerance systems are sufficiently activated.

Various countermeasures have been developed to reduce losses of yield and quality during heat waves. Indoor farming systems are available to control growth temperatures [20]. Breeding can produce thermotolerant crops. Although these tactics are effective, they are too costly and time-consuming to easily apply in many cases. Moreover, the cultivation of crops produced by molecular breeding, including genetic transformation and genome editing technologies, has not completely obtained acceptance from society [21]. Recently, biostimulants that can enhance the growth of plants and ameliorate their stress symptoms have been practically used in agriculture [22-24]. However, few biostimulants designed to enhance the heat tolerance of crops have been developed. This review describes the beneficial effects of essential oil compounds to plants, especially activities that enhance 
heat tolerance. Mechanisms for ameliorating heat damage and their possible application to agriculture are also discussed. In this paper, chemicals that increase the heat adaptability of plants are designated as heat tolerance enhancers (HTLEs).

\section{HTLEs as biostimulants}

It is known that biostimulants consist of biosubstances and microorganisms that enhance crop yield and quality $[25,26]$. They are produced from humic substances, protein hydrolysates, seaweeds, land plants, chitosan, inorganic compounds, and beneficial microorganisms [24]. Biostimulants alleviate abiotic stresses in crop production. It has been reported that a large number of biostimulants could enhance the drought, salinity, and cold tolerance of plants [26]. In contrast, limited examples of biostimulants (e.g. Pseudomonas putida, protein hydrolysates, and seaweed extracts) have been shown to elevate the heat tolerance of crops [26]. Thus, studies on HTLEs promote the development of efficient biostimulants to reduce heat damage to crops.

\subsection{Physical response to heat shock}

Plants respond to heat with various symptoms, such as membrane fluidization, protein denaturation, enzyme inactivation, metabolic disturbances, photosynthesis inhibition, and reactive oxygen species (ROS) generation [27-29]. Heat-exposed plants accumulate compatible solutes, enhance antioxidative activities, and express heat shock proteins (HSPs) [30, 31]. HSP expression is a major response to elevated temperature for many organisms. HSPs are molecular chaperones that can prevent the heat denaturation of proteins. They have different classes, such as HSP60s, HSP70s, HSP90s, HSP100s, and small HSPs characterized according to molecular weight. As HSPs protect proteins from denaturation, organisms including plants can tolerate high temperatures when HSPs are highly expressed $[27,32,33]$. Indeed, heat tolerance has been shown to be enhanced by the overexpression of HSP genes in transgenic plants [34-36]. Mutants exhibiting reduced expression of $H S P$ genes have been reported to show hypersensitivity to high ambient temperatures [37, 38]. This means that substances enhancing the expression of HSP genes are promising biostimulants for ameliorating heat damage to crops.

\subsection{HTLES}

Although elevation of ambient temperature is the most effective cue for the induction of HSP genes [i.e. heat shock response (HSR)], some phytochemicals also induce apparent HSR in plants. It has been found that salicylic acid [39], benzyl alcohol [40], celastrol [41], isothiocyanates [11], sanguinarine [42], monoterpenes [12], and (E)-2-hexenal [13] induce HSR in plants, indicating that these chemicals are HTLEs. Plant-derived HSR inducers that have been identified by using plant systems are summarized in Table 1. In most cases, the mechanisms underlying HSR induction by phytochemical HTLEs have not been elucidated. However, the HSR-inducing mechanism of geldanamycin (GDA) in the Arabidopsis thaliana (L.) Heynh. system has been proposed [44]. GDA, a benzoquinone ansamycin antitumor antibiotic, is an inhibitor of HSP90 [45]. In Arabidopsis cells under normal conditions, HSP90 holds and inactivates a heat shock factor (HSF), a transcription factor for the induction of HSR. GDA inhibits HSP90 via its ATP-binding pocket and facilitates the release of HSF from HSP90. This can trigger HSF activation, after which HSR occurs. Moreover, various HSP90 inhibitors other than GDA could also induce the

Table 1: Plant-derived HSR inducers in plant systems.

\begin{tabular}{lllr}
\hline Compound & Chemical group & Tested plant & Reference \\
\hline Sanguinarine & Alkaloids & Arabidopsis thaliana (L.) Heynh. & [42] \\
Salicylic acid & Aromatics & Arabidopsis thaliana (L.) Heynh. & [39] \\
Benzyl alcohol & Aromatics & Physcomitrella patens (Hedw.) B.S.G. & {$[40]$} \\
(S)-Perillaldehyde & Monoterpenes & Arabidopsis thaliana (L.) Heynh. & {$[12]$} \\
d-Limonene & Monoterpenes & Arabidopsis thaliana (L.) Heynh. & {$[12]$} \\
Celastrol & Triterpenes & Physcomitrella patens (Hedw.) B.S.G. \\
Phenethyl isothiocyanate & Isothiocyanates & Arabidopsis thaliana (L.) Heynh. & {$[41]$} \\
Isopropyl isothiocyanate & Isothiocyanates & Arabidopsis thaliana (L.) Heynh. & {$[11]$} \\
(E)-2-Hexenal & Carbonyls & Arabidopsis thaliana (L.) Heynh. & {$[43]$} \\
\hline
\end{tabular}


HSR of Arabidopsis [46], suggesting that HSP90 is likely a major regulator of the HSR. Recently, the benzophenanthridine alkaloid sanguinarine was predicted to have a similar mechanism to GDA. When GDA and sanguinarine were added to Arabidopsis plants, similar dose and timecourse responses were observed in the gene expression and protein accumulation of HSPs [42, 47]. GDA and sanguinarine inhibited luciferase folding by wheat (Triticum aestivum L.) germ chaperones at similar efficiencies [47]. This suggests that sanguinarine induces HSR probably via the inhibition of chaperones that hold HSF [48]. However, it is unclear how essential oil HTLEs such as isothiocyanates and monoterpenes promote HSR in plants. In the following section, the features of essential oil HTLEs are described in detail.

\section{Isothiocyanates}

Isothiocyanates are sulfur-containing volatile compounds. The common chemical formula is $\mathrm{R}-\mathrm{N}=\mathrm{C}=\mathrm{S}$, in which $\mathrm{N}=\mathrm{C}=\mathrm{S}$ is the isothiocyanate group. More than 100 isothiocyanates have been identified from Brassicaceae, Capparaceae, and Caricaceae plants, whereas Brassicaceae is the major producing family [49]. Isothiocyanates are produced from thioglucosides (glucosinolates) by a specific thioglucosidase (myrosinase, EC 3.2.3.1). As glucosinolates and myrosinases are localized in different cellular and subcellular compartments, the emission of isothiocyanates by plants is kept at low levels [50, 51]. Once cells are mechanically damaged, glucosinolates and myrosinase can access each other, and then isothiocyanates are produced. This reaction is called the glucosinolate-myrosinase system [52]. As typical isothiocyanates are pungent, plants producing them are used as condiments, including mustard (Brassica juncea L.), wasabi (Wasabia japonica Matsum.), and watercress (Nasturtium officinale R. Br.). Moreover, the physiological functions of isothiocyanates in plants have been studied. The emission of isothiocyanates is thought to be a defense system of plants producing glucosinolates, because isothiocyanates inhibit the growth of microorganisms and repel herbivores [52]. Isothiocyanates also affect the growth of plants: they inhibit seed germination and retard plant growth. Aromatic isothiocyanates show more severe growth inhibition than aliphatic isothiocyanates [53]. Growth inhibition by isothiocyanates might be due to interference with cellular functions. Allyl isothiocyanate inhibits the movement of filaments and actin-dependent intracellular transport in Arabidopsis [54].
Traditionally, Brassicaceae plants have been used as green manures to improve crops and inhibit weeds [55]. The effects of Brassicaceae green manures are thought to be based on the antimicrobial, animal-repellent, and allelopathic activities of isothiocyanates. In recent studies, however, isothiocyanates showed positive effects on the stress tolerance of plants at concentrations lower than herbicidal doses. When about $1 \mathrm{mM}$ of isothiocyanates was administered to Arabidopsis, the expression of HSPs and the heat tolerance of the plants were enhanced [11], suggesting that isothiocyanates acted as HTLEs in the plants. However, isothiocyanates at higher concentrations (10-100 mM) killed the plants by inducing oxidative burstlike responses, such as hydrogen peroxide generation and leaf bleaching [56]. Moreover, the levels of HSR-inducing activities differed among isothiocyanates: the activities of aliphatic isothiocyanates were stronger than those of aromatic isothiocyanates [43]. The strongest inducer of HSR was isopropyl isothiocyanate. This indicates that choosing both the appropriate molecular species and their concentrations is crucial for the use of isothiocyanates as HTLEs. Other studies indicated that allyl isothiocyanate induced stomatal closure and upregulated glutathione $S$-transferases, which are detoxifying enzymes, suggesting that isothiocyanate may protect plants from damage caused by environmental stresses [56, 57]. Brassicaceae green manures are biostimulants from which diverse effects (i.e. antimicrobial, animal-repellent, allelopathic, and stress-ameliorating activities) are expected.

\section{Monoterpenes}

Monoterpenes consisting of C10-isoprenoid structures are major components of essential oils produced by plants. The basic structures of monoterpenes are biosynthesized from geranyl diphosphate and/or neryl diphosphate [58]. Highly diverse structures are organized by additional rearrangements and oxidations. As monoterpenes have characteristic flavors, they have been used as components of food flavorings, folk medicines, and cosmetics [5]. Monoterpenes have many physiological functions, such as health-protective (e.g. antioxidative, anti-inflammatory, and chemoprotective activities) [4] and chemicalecological functions (e.g. antibacterial, insecticidal, and repellent activities) $[5,6]$. They are known to affect plant growth. Many reports have examined their allelopathic and herbicidal activities. Myrcene and ocimene induce the expression of stress- or defense-related genes of Arabidopsis [59]. Some monoterpenes severely damage plant cells 
by inducing apoptosis-like cell death [60]. Besides such growth-inhibition activities, monoterpenes have been implicated in stress tolerance, especially thermotolerance. The heat tolerance of Quercus species is enhanced by exogenous monoterpenes [61, 62], probably due to their membrane stabilization activities and ROS scavenging activities [61, 63]. As the emission of monoterpenes by Quercus ilex L. is promoted by heat stress, monoterpenes are likely chemical messengers that transduce heat signals from plants to other plants. Heat-induced monoterpene emission has also been observed in Solanum lycopersicum L. [64] and Musa species [63]. Recently, it was reported that monoterpenes such as d-limonene, $(S)$ perillaldehyde, d-camphor, (-)-borneol, 1,8-cineol, and $\alpha$-pinene induce the HSR of Arabidopsis. Therefore, these monoterpenes are HTLEs [12]. This suggests that the heattolerance-enhancing activities of monoterpenes in plants might be attributable partially to the promotion of HSP accumulation. It is noteworthy that the fumigant used to enhance the heat tolerance of $Q$. ilex contains d-limonene and $\alpha$-pinene, which were found to induce HSR in the Arabidopsis system [61, 65].

As monoterpenes show antibacterial, insecticidal, and repellent activities, plant extracts rich in monoterpenes can be used as biostimulants to reduce pathogenic and feeding damage to crops. In addition, biostimulants containing monoterpene HTLEs can reduce heat-related crop damage. Although $(S)$-perillaldehyde and d-limonene are potent HSR inducers, they have rather narrow ranges effective for HSR-inducing activities [12], suggesting that these monoterpenes are not suitable for use as biostimulants to enhance heat tolerance. Conversely, d-camphor, (-)-borneol, 1,8-cineol, and $\alpha$-pinene are possible components of biostimulants as HTLEs because these monoterpenes have wide effective doses for HSR-inducing activities [12].

\section{Others}

Reactive short-chain leaf volatiles induce the HSP genes of Arabidopsis [13]. The effective volatiles are (E)-2-hexenal and (E)-2-butenal. They are $\alpha, \beta$-unsaturated carbonyls, which are produced from polyunsaturated fatty acids by peroxidation. Benzene derivatives (a series of chlorophenols, benzyl alcohol, and salicylate) activate the heat shock promoter of moss Physcomitrella patens (Hedw.) B.S.G. [41]. As these chemicals can be categorized as HTLEs, plant residues and extracts containing these chemicals can be used as biostimulants.

\section{Action mechanisms of essential oil HTLEs}

The comprehensive mechanisms of chemical HSR inducers have not been confirmed. Benzyl alcohol, which has been traditionally used to induce HSR in some organisms, was believed to enhance membrane fluidity in cells [66]. In a previous study, high concentrations of benzyl alcohol (e.g. $>10 \mathrm{mM}$ ) were needed to induce efficient HSR [67]. However, the effective concentrations of essential oil HTLEs, such as isothiocyanates and monoterpenes, were apparently lower than that of benzyl alcohol, suggesting that the action mechanisms of isothiocyanates and monoterpenes were different from that of benzyl alcohol.

As GDA and sanguinarine efficiently inhibit wheat chaperones at micromolar concentrations, these compounds have been designated as chaperoneinhibition-type HTLEs. However, (S)-perillaldehyde is not categorized as a chaperone-inhibition-type HTLE because the monoterpene weakly inhibits wheat chaperones at millimolar levels [12]. Moreover, isothiocyanates also show slight inhibition of wheat chaperones at millimolar concentrations (our unpublished results). Accordingly, (S)-perillaldehyde and isothiocyanates should be designated as non-chaperone-inhibition-type HTLEs. As (S)-perillaldehyde and isothiocyanates are known to induce ROS production in cells $[56,68]$, such HTLEs may induce HSR via ROS signaling (Figure 1). Further studies are required to confirm the HSR-inducing mechanisms of nonchaperone-inhibition-type HTLEs.

\section{Practical uses of essential oil HTLEs}

As essential oils such as isothiocyanates and monoterpenes have allelopathic, antimicrobial, insecticidal, and repellent activities, biostimulants containing these compounds are expected to reduce the damages by weeds, pathogens, and herbivores. In addition, some monoterpenes and isothiocyanates enhance the heat tolerance of plants by inducing the expression of HSP genes. HSPs are related to not only heat tolerance but also diverse stress tolerances, suggesting that the high expression state of HSPs provided by essential oil HTLEs enables plants to tolerate various stresses (Figure 2). If that is the case, biostimulants containing essential oil HTLEs may be able 


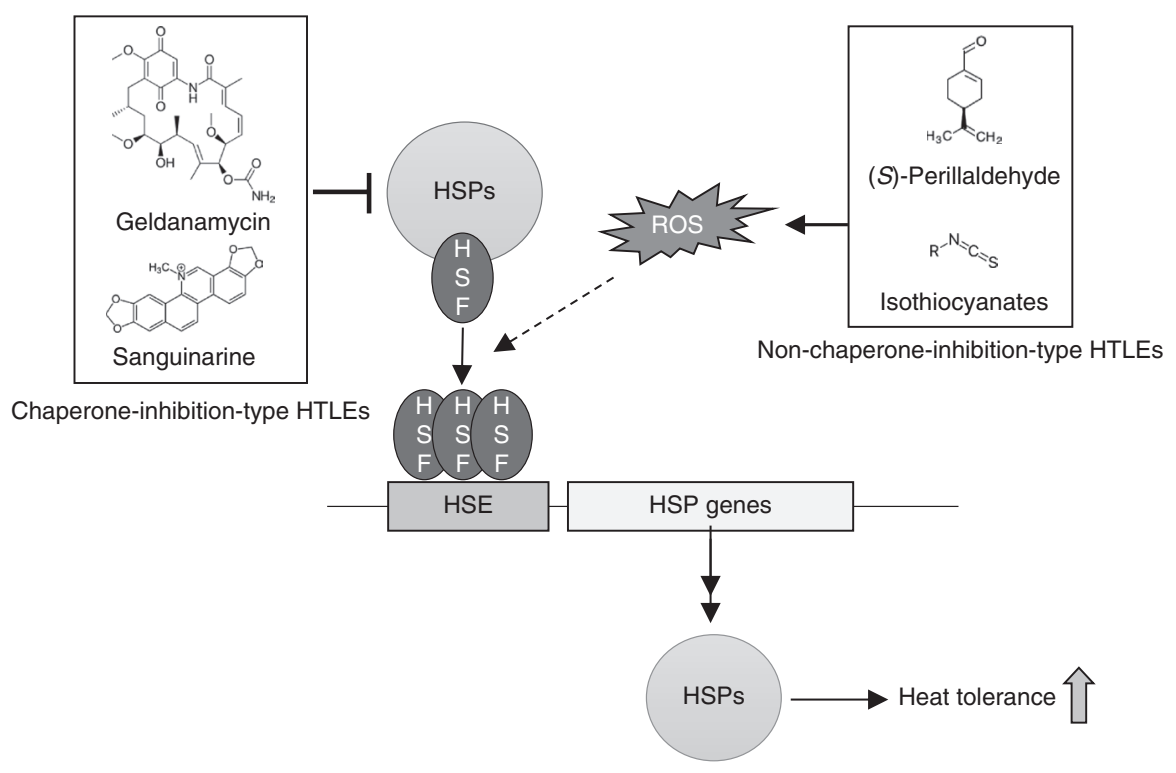

Figure 1: Putative action mechanisms of essential oil HTLEs. Geldanamycin and sanguinarine are chaperone-inhibition-type HTLEs. (S)-perillaldehyde and isothiocyanates are thought to be non-chaperone-inhibition-type HTLEs. Chaperone-inhibition-type HTLEs may release HSF by inhibiting HSPs. Non-chaperone-inhibition-type HTLEs may activate HSF via ROS generation. Activated HSFs promote the expression of HSP genes, and then the heat tolerance of plants is enhanced.

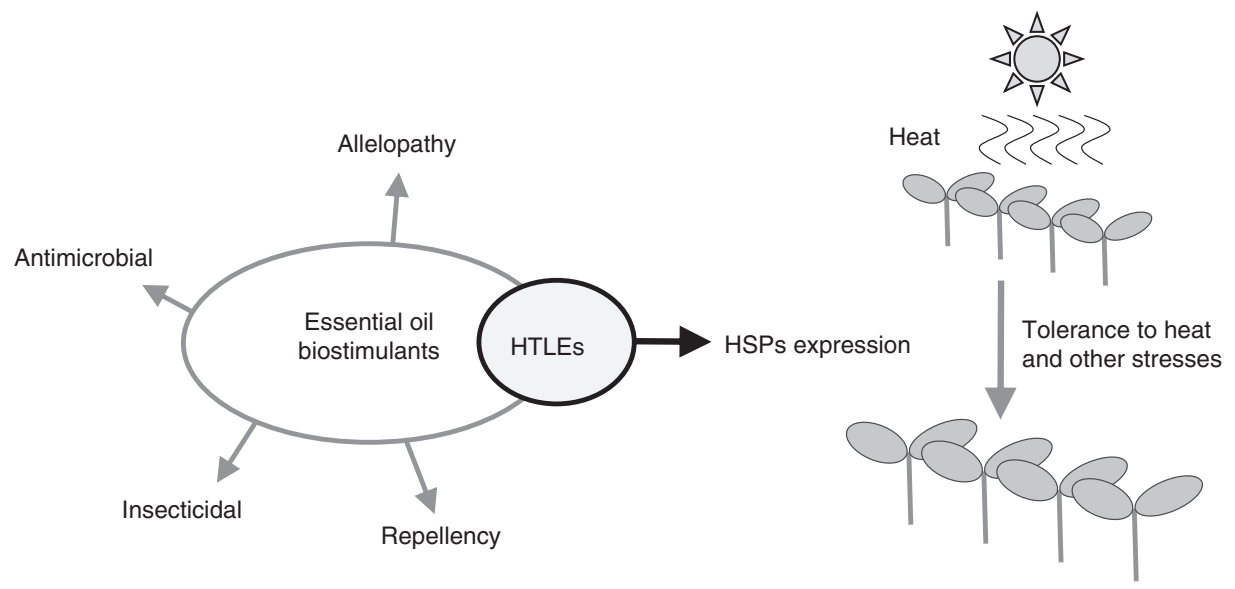

Figure 2: Diverse effects of biostimulants that contain essential oils on the production of crops. Essential oil HTLEs may facilitate the growth of plants under high-temperature conditions.

to ameliorate multiple kinds of damage to crops. Moreover, natural essential oils will be easily accepted by consumers, because essential oils have been used for a long period in human history. If activity, safety, and stability conditions are established, essential oil HTLEs would be widely commercialized in the future. However, it should be noted that quantitative administration to crops would be difficult because most essential oils are composed of volatile compounds. The development of a dose-control system is needed for the practical use of essential oil HTLEs in agriculture.

\section{Conclusion}

Essential oils such as isothiocyanates and monoterpenes enhance the heat tolerance of plants. Biostimulants containing essential oil HTLEs may be useful for reducing losses of crop yield and quality due to various stresses, especially heat. However, physiological responses for essential oil HTLEs have been tested only in model plants, i.e. A. thaliana and P. patens. Therefore, practical studies on essential oil HTLEs are needed. Although a great deal of biostimulants have been developed, few have 
demonstrated heat-tolerance-enhancing activities. Intensive investigations of HSR-inducing activities will uncover biostimulants that can enhance the heat tolerance of crops. Moreover, large-scale screening may provide more effective essential oil HTLEs that are useful for enhancing the functions of biostimulants. For the use of essential oil HTLEs, the chemical species and application methods should be determined according to the species and growth stages of crops. The ecological and environmental influence should be investigated. Further studies will deepen our understanding of essential oil HTLEs and facilitate their practical use as biostimulants.

\section{References}

1. Burt S. Essential oils: their antibacterial properties and potential applications in foods - a review. Int J Food Microbiol 2004;94:223-53.

2. Bakkali F, Averbeck S, Averbeck D, Idaomar M. Biological effects of essential oils - a review. Food Chem Toxicol 2008;46:446-75.

3. Raut JS, Karuppayil SM. A status review on the medicinal properties of essential oils. Ind Crop Prod 2014;62:250-64.

4. Dhifi W, Bellili S, Jazi S, Bahloul N, Mnif W. Essential oils' chemical characterization and investigation of some biological activities: a critical review. Medicines 2016;3:25.

5. Kalemba D, Kunicka A. Antibacterial and antifungal properties of essential oils. Curr Med Chem 2003;10:813-29.

6. Nerio LS, Olivero-Verbel J, Stashenko E. Repellent activity of essential oils: a review. Biores Technol 2010;101:372-8.

7. Nazzaro F, Fratianni F, De Martino L, Coppola R, De Feo V. Effect of essential oils on pathogenic bacteria. Pharmaceuticals 2013;6:1451-74.

8. Dudai N, Poljakoff-Mayber A, Mayer AM, Putievsky E, Lerner HR. Essential oils as allelochemicals and their potential use as bioherbicides. J Chem Ecol 1999;25:1079-89.

9. Tworkoski T. Herbicide effects of essential oils. Weed Sci 2002;50:425-31.

10. Koul O, Walia S, Dhaliwal GS. Essential oils as green pesticides: potential and constraints. Biopest Int 2008;4:63-84.

11. Hara M, Harazaki A, Tabata K. Administration of isothiocyanates enhances heat tolerance in Arabidopsis thaliana. Plant Growth Regul 2013;69:71-7.

12. Hara M, Yamauchi N, Sumita Y. Monoterpenes induce the heat shock response in Arabidopsis. Z Naturforsch C 2018;73:177-84.

13. Yamauchi Y, Kunishima M, Mizutani M, Sugimoto Y. Reactive short-chain leaf volatiles act as powerful inducers of abiotic stress-related gene expression. Sci Rep 2015;5:8030.

14. Schmidhuber J, Tubiello FN. Global food security under climate change. Proc Natl Acad Sci USA 2007;104:19703-8.

15. Lobell DB, Schlenker W, Costa-Roberts J. Climate trends and global crop production since 1980. Science 2011;333:616-20.

16. Challinor AJ, Watson J, Lobell DB, Howden SM, Smith DR, Chhetri N. A meta-analysis of crop yield under climate change and adaptation. Nat Clim Change 2014;4:287.

17. Ainsworth EA, Ort DR. How do we improve crop production in a warming world? Plant Physiol 2010;154:526-30.
18. Teskey R, Wertin T, Bauweraerts I, Ameye M, McGuire MA, Steppe K. Responses of tree species to heat waves and extreme heat events. Plant Cell Environ 2015;38:1699-712.

19. Larcher W. Physiological plant ecology, 4th ed. Berlin, Heidelberg: Springer, 2003.

20. Morimoto T, Torii T, Hashimoto Y. Optimal control of physiological processes of plants in a green plant factory. Control Eng Pract 1995;3:505-11.

21. Ishii T, Araki M. Consumer acceptance of food crops developed by genome editing. Plant Cell Rep 2016;35:1507-18.

22. Calvo P, Nelson L, Kloepper JW. Agricultural uses of plant biostimulants. Plant Soil 2014;383:3-41.

23. Du Jardin P. Plant biostimulants: definition, concept, main categories and regulation. Sci Hort 2015;196:3-14.

24. Yakhin OI, Lubyanov AA, Yakhin IA, Brown PH. Biostimulants in plant science: a global perspective. Front Plant Sci 2017;7:2049.

25. Vargas-Hernandez M, Macias-Bobadilla I, Guevara-Gonzalez RG, Romero-Gomez SJ, Rico-Garcia E, Ocampo-Velazquez RV, et al. Plant hormesis management with biostimulants of biotic origin in agriculture. Front Plant Sci 2017;8:1762.

26. Van Oosten MJ, Pepe O, De Pascale S, Silletti S, Maggio A. The role of biostimulants and bioeffectors as alleviators of abiotic stress in crop plants. Chem Biol Technol Agric 2017;4:5.

27. Wahid A, Gelani S, Ashraf M, Foolad MR. Heat tolerance in plants: an overview. Environ Exp Bot 2007;61:199-223.

28. Allakhverdiev SI, Kreslavski VD, Klimov VV, Los DA, Carpentier R, Mohanty P. Heat stress: an overview of molecular responses in photosynthesis. Photosynth Res 2008;98:541.

29. Ruelland E, Zachowski A. How plants sense temperature. Environ Exp Bot 2010;69:225-32.

30. Iba K. Acclimative response to temperature stress in higher plants: approaches of gene engineering for temperature tolerance. Annu Rev Plant Biol 2002;53:225-45.

31. Kotak S, Larkindale J, Lee U, von Koskull-Döring P, Vierling E, Scharf KD. Complexity of the heat stress response in plants. Curr Opin Plant Biol 2007;10:310-6.

32. Wang W, Vinocur B, Shoseyov 0, Altman A. Role of plant heatshock proteins and molecular chaperones in the abiotic stress response. Trends Plant Sci 2004;9:244-52.

33. Waters ER. The evolution, function, structure, and expression of the plant sHSPs. J Exp Bot 2013;64:391-403.

34. Zhang L, Zhang Q, Gao Y, Pan H, Shi S, Wang Y. Overexpression of heat shock protein gene PfHSP21.4 in Arabidopsis thaliana enhances heat tolerance. Acta Physiol Plant 2014;36:1555-64.

35. Sung DY, Guy CL. Physiological and molecular assessment of altered expression of Hsc70-1 in Arabidopsis. Evidence for pleiotropic consequences. Plant Physiol 2003;132:979-87.

36. Masand S, Yadav SK. Overexpression of MuHSP70 gene from Macrotyloma uniflorum confers multiple abiotic stress tolerance in transgenic Arabidopsis thaliana. Mol Biol Rep 2016;43:53-64.

37. Su PH, Li HM. Arabidopsis stromal 70-kD heat shock proteins are essential for plant development and important for thermotolerance of germinating seeds. Plant Physiol 2008;146:1231-41.

38. Leng L, Liang Q, Jiang J, Zhang C, Hao Y, Wang X, et al. A subclass of HSP70s regulate development and abiotic stress responses in Arabidopsis thaliana. J Plant Res 2017;130:349-63.

39. Clarke SM, Mur LA, Wood JE, Scott IM. Salicylic acid dependent signaling promotes basal thermotolerance but is not essential for acquired thermotolerance in Arabidopsis thaliana. Plant J 2004;38:432-47. 
40. Saidi Y, Finka A, Chakhporanian M, Zrÿd JP, Schaefer DG, Goloubinoff P. Controlled expression of recombinant proteins in Physcomitrella patens by a conditional heat-shock promoter: a tool for plant research and biotechnology. Plant Mol Biol 2005;59:697-711.

41. Saidi Y, Domini M, Choy F, Zryd JP, Schwitzguebel JP, Goloubinoff $P$. Activation of the heat shock response in plants by chlorophenols: transgenic Physcomitrella patens as a sensitive biosensor for organic pollutants. Plant Cell Environ 2007;30:753-63.

42. Hara M, Kurita I. The natural alkaloid sanguinarine promotes the expression of heat shock protein genes in Arabidopsis. Acta Physiol Plant 2014;36:3337-43.

43. Hara M, Matsubara T, Takahashi I, Murano H. Isobutyl isothiocyanate is a potent heat tolerance enhancer for Arabidopsis. Environ Control Biol 2018;56:121-6.

44. Yamada K, Fukao Y, Hayashi M, Fukazawa M, Suzuki I, Nishimura M. Cytosolic HSP90 regulates the heat shock response that is responsible for heat acclimation in Arabidopsis thaliana. J Biol Chem 2007;282:37794-804.

45. Roe SM, Prodromou C, O’Brien R, Ladbury JE, Piper PW, Pearl LH. Structural basis for inhibition of the Hsp90 molecular chaperone by the antitumor antibiotics radicicol and geldanamycin. J Med Chem 1999;42:260-6.

46. Murano H, Matsubara T, Takahashi I, Hara M. A purine-type heat shock protein 90 inhibitor promotes the heat shock response in Arabidopsis. Plant Biotechnol Rep 2017;11:107-13.

47. Matsuoka E, Matsubara T, Takahashi I, Murano H, Hara M. The isoquinoline alkaloid sanguinarine which inhibits chaperone activity enhances the production of heat shock proteins in Arabidopsis. Plant Biotechnol 2016;33:409-13.

48. Matsuoka E, Kato N, Hara M. Induction of the heat shock response in Arabidopsis by heat shock protein 70 inhibitor VER155008. Funct Plant Biol 2019;46:925-32.

49. Fahey JW, Zalcmann AT, Talalay P. The chemical diversity and distribution of glucosinolates and isothiocyanates among plants. Phytochemistry 2001;56:5-51.

50. Bones AM, Rossiter JT. The myrosinase-glucosinolate system, its organisation and biochemistry. Physiol Plant 1996;97: 194-208.

51. Ishida M, Hara M, Fukino N, Kakizaki T, Morimitsu Y. Glucosinolate metabolism, functionality and breeding for the improvement of Brassicaceae vegetables. Breed Sci 2014;64:48-59.

52. Halkier BA, Gershenzon J. Biology and biochemistry of glucosinolates. Annu Rev Plant Biol 2006;57:303-33.

53. Urbancsok J, Bones A, Kissen R. Glucosinolate-derived isothiocyanates inhibit Arabidopsis growth and the potency depends on their side chain structure. Int I Mol Sci 2017;18:2372.

54. Sporsheim B, Øverby A, Bones A. Allyl isothiocyanate inhibits actin-dependent intracellular transport in Arabidopsis thaliana. Int J Mol Sci 2015;16:29134-47.
55. Jabran K. Brassicaceae allelopathy for weed control. In: Manipulation of allelopathic crops for weed control. Cham, Germany: Springer, 2017:21-27.

56. Hara M, Yatsuzuka Y, Tabata K, Kuboi T. Exogenously applied isothiocyanates enhance glutathione $S$-transferase expression in Arabidopsis but act as herbicides at higher concentrations. J Plant Physiol 2010;167:643-9.

57. Khokon MA, Jahan MS, Rahman T, Hossain MA, Muroyama D, Minami I, et al. Allyl isothiocyanate (AITC) induces stomatal closure in Arabidopsis. Plant Cell Environ 2011;34:1900-6.

58. Dudareva N, Pichersky E, Gershenzon J. Biochemistry of plant volatiles. Plant Physiol 2004;135:1893-902.

59. Godard KA, White R, Bohlmann J. Monoterpene-induced molecular responses in Arabidopsis thaliana. Phytochemistry 2008;69:1838-49.

60. Ghosh S, Singh UK, Meli VS, Kumar V, Kumar A, Irfan M, et al. Induction of senescence and identification of differentially expressed genes in tomato in response to monoterpene. PLoS One 2013;8:e76029.

61. Peñuelas J, Llusià J. Linking photorespiration, monoterpenes and thermotolerance in Quercus. New Phytol 2002;155:227-37.

62. Delfine S, Csiky O, Seufert G, Loreto F. Fumigation with exogenous monoterpenes of a non-isoprenoid-emitting oak (Quercus suber): monoterpene acquisition, translocation, and effect on the photosynthetic properties at high temperatures. New Phytol 2000;146:27-36.

63. Jardine KJ, Jardine AB, Holm JA, Lombardozzi DL, Negron-Juarez RI, Martin ST, et al. Monoterpene 'thermometer' of tropical forest-atmosphere response to climate warming. Plant Cell Environ 2017;40:441-52.

64. Copolovici L, Kännaste A, Pazouki L, Niinemets U. Emissions of green leaf volatiles and terpenoids from Solanum lycopersicum are quantitatively related to the severity of cold and heat shock treatments. J Plant Physiol 2012;169:664-72.

65. Loreto F, Forster A, Durr M, Csiky O, Seufert G. On the monoterpene emission under heat stress and on the increased thermotolerance of leaves of Quercus ilex L. fumigated with selected monoterpenes. Plant Cell Environ 1998;21:101-7.

66. Vigh L, Maresca B, Harwood JL. Does the membrane's physical state control the expression of heat shock and other genes? Trends Biochem Sci 1998;23:369-74.

67. Sangwan V, Örvar BL, Beyerly J, Hirt H, Dhindsa RS. Opposite changes in membrane fluidity mimic cold and heat stress activation of distinct plant MAP kinase pathways. Plant J 2002;31:629-38.

68. Tian J, Pan C, Zhang M, Gan YY, Pan SY, Liu M, et al. Induced cell death in Ceratocystis fimbriata by pro-apoptotic activity of a natural organic compound, perillaldehyde, through $\mathrm{Ca}^{2+}$ overload and accumulation of reactive oxygen species. Plant Pathol 2019;68:344-7. 